\title{
Cutaneous manifestations of autoinflammatory diseases
}

Di Wu ${ }^{1}$, Min Shen ${ }^{1, *}$, Qingping Yao ${ }^{2, *}$

${ }^{1}$ Department of Rheumatology and Clinical Immunology, State Key Laboratory of Complex Severe and Rare Diseases, Peking Union Medical College Hospital, Chinese Academy of Medical Sciences and Peking Union Medical College, National Clinical Research Center for Dermatologic and Immunologic Diseases (NCRC-DID), Key Laboratory of Rheumatology and Clinical Immunology, Ministry of Education, Beijing, China ${ }^{2}$ Division of Rheumatology, Allergy, and Immunology, Stony Brook University School of Medicine, Stony Brook, NY, USA

Abstract

Received July 16, 2021 accepted October 07, 2021

Autoinflammatory diseases (AIDs) are a heterogeneous group of disorders in which recurrent or continuous aseptic inflammation arises primarily through antigen-independent hyperactivation of the innate immune system. The skin is frequently involved with a wide variety of cutaneous manifestations, most of which are non-specific. Recognition of skin lesions in AIDs may sometimes provide clues for a correct diagnosis. In this review, the cutaneous involvements of $>20$ selected AIDs were summarized and organized into different categories based on their characteristic manifestations, such as urticarial dermatosis, neutrophilic dermatosis, granulomatosis, chilblain, lipodystrophy, and hyperkeratosis. With this classification scheme, cutaneous manifestations in AIDs could be more easily identified to facilitate diagnosis in clinical practice.

Keywords

autoinflammatory diseases $\cdot$ urticarial dermatosis $\cdot$ neutrophilic dermatosis $・$ granulomatosis $\cdot$ chilblain $\cdot$ lipoatrophy -keratinization diseases

\section{Introduction}

Autoinflammatory diseases (AIDs) are a group of medical disorders in which recurrent or continuous pathogenic inflammation arises primarily through antigen-independent hyperactivation of immune pathways, derived from defects or dysregulation of the innate immune system..$^{[1,2]}$ Since the introduction of AIDs in 1999, >30 monogenic diseases and some polygenic or multifactorial diseases have been classified among this clinical group. ${ }^{[3]}$ The skin is involved in many AIDs, with a wide variety of cutaneous manifestations, which can be non-specific but sometimes can be the key to a correct diagnosis. In this review, we summarize the cutaneous lesions of main AIDs (Table 1) using a recently proposed dermatological classification system based on characteristic skin manifestations. ${ }^{[4]}$

\section{Autoinflammatory Urticarial Dermatosis}

The urticarial rash is a kind of skin lesion with red, raised, and mostly itchy wheal. ${ }^{[4]}$ Individual rash lasts for a few hours to days and resolves without other skin changes. Symptoms and flares in the group of autoinflammatory urticarial dermatosis are often triggered by cold or emotional stress..$^{[5]}$ The presence of chronic urticaria in infants, especially triggered by cold, is a cue for AIDs.

\section{NLRP3-associated autoinflammatory disease (NLRP3- AID)}

NLRP3-AID, also known as a cryopyrin-associated periodic syndrome (CAPS), ${ }^{[2]}$ is a group of autosomal dominant diseases characterized by inflammation caused by a mutation

\footnotetext{
Address for correspondence:

"Min Shen, Department of Rheumatology and Clinical Immunology, Peking

Union Medical College Hospital, No. 1 Shuaifuyuan, Dongcheng District, Beijing

100730, China. E-mail: shenmpumch@163.com.

'Qingping Yao, Division of Rheumatology, Allergy and Immunology, Stony Brook

University, HSC Tower 040, Stony Brook, NY 11794, USA.

E-mail: Qingping.yao@stonybrookmedicine.edu
} 
Table 1: Cutaneous manifestations of main AIDs

\begin{tabular}{|c|c|c|c|}
\hline Cutaneous manifestation & Disease & Gene & Systems-based classification $^{[23]}$ \\
\hline \multirow[t]{5}{*}{ Urticarial dermatosis } & $\begin{array}{l}\text { NLRP3-associated autoinflammatory dis- } \\
\text { ease (NLRP3-AID) }\end{array}$ & $N L R P 3$ & Inflammasomopathy \\
\hline & $\begin{array}{l}\text { NLRP12-associated autoinflammatory } \\
\text { disease (NLRP12-AID) }\end{array}$ & NLRP12 & Inflammasomopathy \\
\hline & $\begin{array}{l}\text { PLCG2 (phospholipase C gamma 2)-as- } \\
\text { sociated antibody deficiency and immune } \\
\text { dysregulation (PLAID) }\end{array}$ & PLCG2 & PLCG2 activation \\
\hline & FCAS4 & $N L R C 4$ & Inflammasomopathy \\
\hline & Schnitzler syndrome & Unknown & Miscellaneous \\
\hline \multirow[t]{5}{*}{ Neutrophilic dermatosis } & $\begin{array}{l}\text { PSTPIP1-associated arthritis, PG, and acne } \\
\text { (PAPA) }\end{array}$ & PSTPIP1 & $\begin{array}{l}\text { Actinopathy (actin cytoskeleton } \\
\text { dysregulation) }\end{array}$ \\
\hline & FMF & MEFV & Inflammasomopathy \\
\hline & PAAND & MEFV & Inflammasomopathy \\
\hline & Haploinsufficiency of A20 (HA20) & TNFAIP3 & NF-KB dysregulation \\
\hline & Majeed syndrome & LPIN2 & IL-1 activation \\
\hline Granulomatosis & BS & NOD2 & NF-KB dysregulation \\
\hline \multirow[t]{3}{*}{ Chilblain } & Familial Chilblain Lupus & TREX1, SAMHD1, STING1 & Interferonopathy \\
\hline & AGS & $\begin{array}{l}\text { TREX1, RNASEH2B, RNASEH2C, } \\
\text { RNASEH2A, SAMHD1, ADAR1, IFIH1 }\end{array}$ & Interferonopathy \\
\hline & SAVI & STING1 & Interferonopathy \\
\hline \multirow[t]{2}{*}{ Lipodystrophy } & PRAAS & PSMB8 & Interferonopathy \\
\hline & ORAS & OTULIN & NF-KB dysregulation \\
\hline \multirow[t]{2}{*}{ Keratinization diseases } & DITRA & IL36RN & IL-36 activation \\
\hline & DIRA & $\operatorname{ILIRN}$ & IL-1 activation \\
\hline \multirow[t]{4}{*}{ Miscellaneous } & MKD & $M V K$ & Inflammasomopathy \\
\hline & TRAPS & TNFRSF1A & $\begin{array}{l}\text { Protein misfolding and endoplasmic } \\
\text { reticulum stress }\end{array}$ \\
\hline & DADA2 & $A D A 2$ & Macrophage activation \\
\hline & YAOS & NOD2 & Miscellaneous \\
\hline
\end{tabular}

AGS, Aicardi-Goutieres syndrome; AIDs, autoinflammatory diseases; BS, Blau syndrome; DADA2, deficiency of adenosine deaminase 2; DIRA, deficiency of the IL-1 receptor antagonist; DITRA, deficiency of the IL-36 receptor antagonist; FCAS, familial cold autoinflammatory syndrome; FMF, familial Mediterranean fever; MKD, mevalonate kinase deficiency; NOD2, nucleotide-binding oligomerization domain containing 2; NLR, nucleotide-binding oligomerization domain-like receptors; ORAS, OTULIN-related autoinflammatory syndrome; PRAAS, proteasome-associated autoinflammatory syndrome; PG, pyoderma gangrenosum; PAAND, Pyrin-associated autoinflammation with neutrophilic dermatosis; SAVI, STING associated vasculopathy with onset in infancy; TRAPS, TNF receptor-associated periodic syndrome; YAOS, Yao syndrome.

in the NLRP3 (nucleotide-binding oligomerization domain-like receptors (NLR) family pyrin domain containing 3 ) gene. ${ }^{[3]}$ Gain-of-function mutations in NLRP3 can cause overactivation of the NLRP3 inflammasome leading to excessive production of the pro-inflammatory cytokines, IL-1 $\beta$ and IL-18, thus, leading to inflammation. The NLRP3-AID spectrum includes three overlapping phenotypes of increasing severity, which were initially designated as a familial cold autoinflammatory syndrome (FCAS), Muckle-Wells syndrome (MWS), and neonatal-onset multisystem inflammatory disease (NOMID) or chronic infantile neurologic cutaneous and articular (CINCA) syndrome. It has been suggested to add the adjectives mild, moderate, and severe phenotypes, instead of using the historical names FCAS, MWS, and CINCA/NOMID, respectively. ${ }^{[2]}$ In most patients, symptoms develop within the first few years of life, although adult-onset cases have been reported ${ }^{[6]}$ The clinical manifestations of NLRP3-AID include systemic features such as fatigue, fever, headache, arthralgia, myalgia, and organ inflammation often presenting as urticarial rash, conjunctivitis, sensorineural hearing loss, and chronic sterile meningitis. ${ }^{[1]}$

The urticarial rash, which is listed as a major symptom in the Eurofever/PRINTO classification criteria for CAPS, ${ }^{[7]}$ affects almost all patients with NLRP3-AID (89\%) and is similar in all three phenotypes. ${ }^{[5]}$ The rashes, especially in FCAS, often appear hours after cold exposure in areas not directly exposed to cold, such as upper arm, trunk, buttocks, or thighs with symmetrical distribution, accompanied by fever, headache, arthralgia, and conjunctivitis. ${ }^{[8,9]}$ The lesions (Figure $1 \mathrm{~A}$ ) are pink or pale red macules or slightly raised papules/plaques, which are usually neither edematous nor annular but may have a peripheral halo of vasoconstriction. The rashes are typically not itchy or with only slight pruritus but are often sensitive to touch. ${ }^{[5,8]}$ Individual lesions 


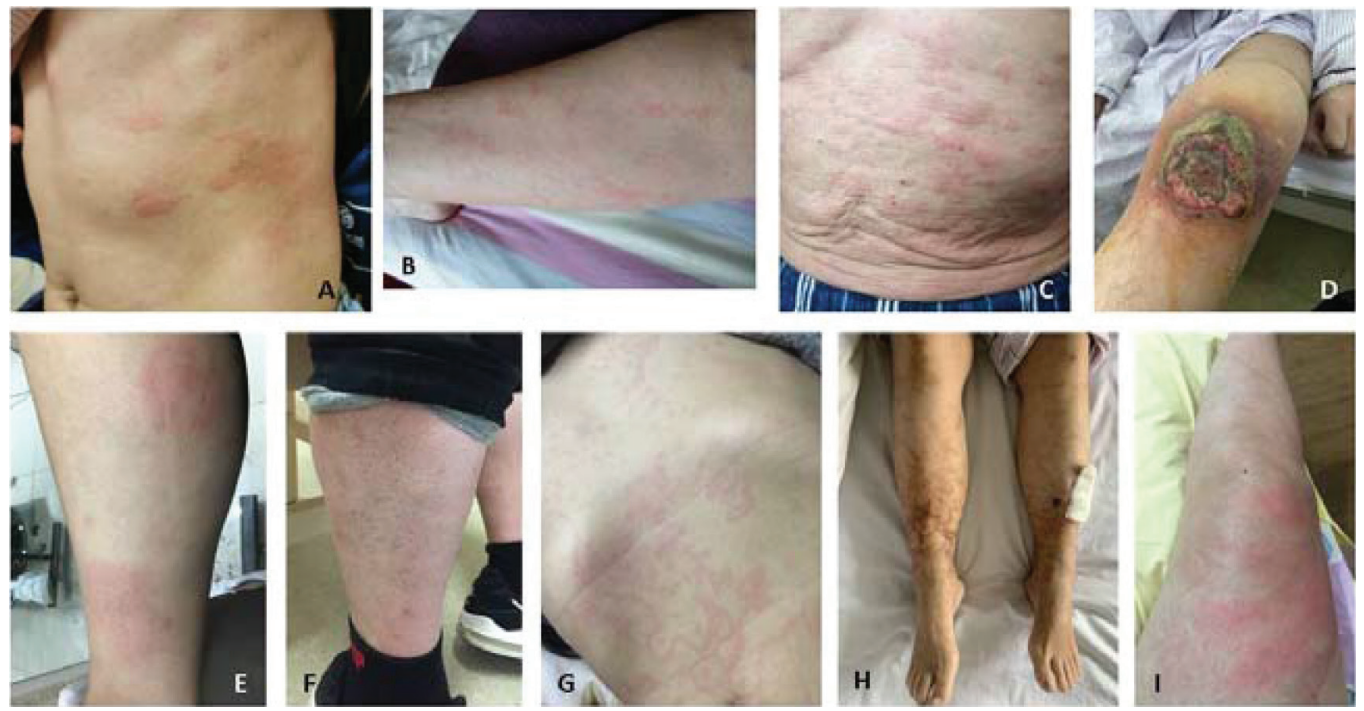

Figure 1: Urticarial rash in NLRP3-AID (A), NLRP12-AID (B) and Schnitzler syndrome (C). PG in PAPA syndrome (D). Erythema nodosumlike lesions in HA20 (E). Ichthyosiform dermatosis in Blau syndrome (BS) (F). Erythematous annular and maculopapular rash in TRAPS (G). Livedo reticularis in DADA2 (H). Erythematous patches in YAOS (I). DADA2, deficiency of adenosine deaminase 2; NLR, nucleotide-binding oligomerization domain-like receptors; PG, pyoderma gangrenosum; TRAPS, TNF receptor-associated periodic syndrome; YAOS, Yao syndrome.

resolve within $24-48 \mathrm{~h}$ without scarring or hyperpigmentation, although more solid and persistent lesions mimicking urticarial vasculitis can also develop. ${ }^{[10]}$ Urticarial rashes in NLRP3-AID are reported to be more flattened and last longer than in spontaneous urticaria, but they can be indistinguishable. Dense interstitial and perivascular neutrophilic infiltrations with no or mild dermal edema can be detected in skin biopsies. ${ }^{[8]}$ Neutrophils are also seen within the epithelia of sweat glands. Leukocytoclasia is frequently observed but fibrinoid necrosis of the dermal small vessel wall is always absent. ${ }^{[10]}$

\section{NLRP12-associated autoinflammatory disease (NLRP12 AID)}

NLRP12-AID is an autosomal dominant disease caused by mutations in the NLRP12 (NLR family pyrin domain containing 12) gene. NLRP12-AID got its former name, familial cold autoinflammatory syndrome 2 (FCAS2), owing to its phenotypic resemblance to the NLRP3-associated FCAS. ${ }^{[1]}$ Pathogenic mutations in NLRP12-AID are generally believed to be loss of function. About $70 \%$ of patients had disease onset in childhood. [11,12] NLRP12-AID is characterized by episodic and recurrent fever, urticarial rash, arthralgia, myalgia, and headache. Recent studies demonstrated that deafness and vision loss are not rare in these patients. ${ }^{[12]}$ Half of the patients reported cold exposure as a trigger for the episodes. Cutaneous involvement in NLRP12-AD includes evanescent pruritic urticarial rash on the trunk, face, and extremities (Figure 1B) in $56 \%$ of patients. ${ }^{[12]}$ Cutis laxa, erythematous malar rash, and oral ulcers have also been reported.

\section{PLCG2 (phospholipase C gamma 2)-associated antibody deficiency and immune dysregulation (PLAID)}

PLAID, also known as FCAS3, ${ }^{[13-15]}$ is an autosomal dominant disorder caused by a genomic deletion in the PLCG2 gene and is characterized by cold urticaria, cutaneous granulomas, humoral immune deficiency, and autoimmune diseases. Mutant PLCG2 increased activation of mast cells is considered responsible for the cold urticaria. ${ }^{[13]}$ Patients developed an erythematous, pruritic urticarial rash and sometimes angioedema within minutes after exposure to cold, such as cold atmosphere, aquatic activities, handling cold objects, and ingestion of cold foods or beverages. ${ }^{[14]}$ Most patients had onset from infancy. Evaporative cooling is a near-universal trigger. Localized cutaneous reactions to water evaporation do not generalize, and most lesions resolve soon after rewarming. Skin lesion biopsy showed mast cell infiltrates with degranulation. Some patients developed blistering rashes at the tip of the nose, ears, and fingers soon after birth. ${ }^{[14]}$ Skin granulomas may appear on the face as tender, red-brown, firm plaques and nodules, eventually leading to skin atrophy. ${ }^{[5]}$

\section{Familial cold autoinflammatory syndrome 4 (FCAS4)}

FCAS4 is an autosomal dominant disorder caused by heterozygous mutation in the NLRC4 (NLR family caspase recruitment domain (CARD) domain containing 4) gene. The gain-of-function mutations in NLRC4 activated caspase-1 leading to increased secretion of IL-1 $\beta .{ }^{[16,17]}$ FCAS4 is characterized by neonatal-onset episodic high fevers, non-itchy urticarial rash, and arthralgia. ${ }^{[17]}$ The symptoms 
were often induced by exposure to cold and resolved without treatment in most cases. Skin biopsies showed a dermal lymphocytic-histiocytic infiltrate rather than neutrophils as in NLRP3-associated FCAS. ${ }^{[16]}$

\section{Schnitzler syndrome}

Schnitzler syndrome is an acquired late-onset (typically in the fifth decade)AID, characterized by two defining features, IgM-k (rarely $\lg G$ ) monoclonal gammopathy, and recurrent urticarial rash. ${ }^{[18]}$ Other common clinical features include recurrent fever, lymphadenopathy, arthralgia, bone pain, fatigue, and systemic inflammatory response. Around $15-20 \%$ of patients evolve into overt lymphoproliferative disease. Although the etiology of Schnitzler syndrome is not completely understood, overproduction of IL-1 $\beta$ has been demonstrated to play an important role. ${ }^{[19]}$ The urticarial rash, usually associated with fever, develops mostly on the trunk and limbs. The eruption consists of erythematous macules or slightly raised papules and plaques and is often more pronounced in the evening hours (Figure 1C). The rash is usually mildly itchy and persists for $<48 \mathrm{~h}$. Angioedema is rarely seen. The rash can be exacerbated by heat or cold exposure, alcohol consumption, stress, or physical exercise. ${ }^{[20]}$ Skin biopsies at a relatively early stage demonstrate a neutrophilic dermal infiltrate without significant dermal edema. Clustering of neutrophils around sweat glands can be observed. ${ }^{[2]}$

\section{Autoinflammatory neutrophilic dermatosis}

A neutrophilic dermatosis is a group of inflammatory skin diseases characterized by aseptic accumulation of neutrophils in the skin. ${ }^{[4]}$ The pathergy phenomenon can be commonly observed and is considered a diagnostic feature.

\section{PSTPIP1-associated arthritis, PG, and acne (PAPA)}

PAPA, also known as pyogenic arthritis, pyoderma gangrenosum, and acne (PAPA) syndrome, is an autosomal dominant disorder caused by mutations in the proline-serine-threonine phosphatase-interacting protein 1 (PSTPIP1) gene. Mutated PSTPIP1 leads to constitutive activation of the pyrin inflammasome, which results in overproduction of IL-1 $1 \beta .^{[1]}$ Clinically, PAPA is characterized by sterile inflammation of the skin and joints. ${ }^{[10]}$ The disease usually starts with deforming pyogenic sterile arthritis in childhood and develops severe cystic acne and pyoderma gangrenosum in adolescence and beyond. ${ }^{[4]}$ Cutaneous manifestations are episodic and recurrent. The most prominent feature is debilitating, aggressive ulcers, usually in the lower extremities, which are often diagnosed as pyoderma gangrenosum (PG) (Figure 1D). PG usually starts as a small erythematous tender papule, pustule, or nodule and then progresses to a painful ulcer that expands rapidly with necrosis and a well-demarcated, elevated, undermined, violaceous border. PG may appear spontaneously or develop after minimal trauma or injection (pathergy phenomenon). ${ }^{[5]}$ The typical histology of PG shows epidermal ulceration and a dermal-hypodermal inflammatory infiltrate mainly consisting of neutrophils, without fibrinoid necrosis of vessel wall. ${ }^{[10]}$ Special histochemical stains and microbiological cultures must be negative. Pustular, bullous, or vegetating lesions can coexist with PG. PG is frequently accompanied by acne, often in its more severe nodulocystic type. Acne rosacea with a prominent pustular component has also been described ${ }^{[5]}$ The phenotypic spectrum of PSTPIP1-associated AIDs has recently been expanded. ${ }^{[21]}$ For example, patients with pyoderma gangrenosum, acne, and suppurative hidradenitis with or without arthritis have been reported as PAPASH syndrome and PASH syndrome, respectively.

\section{FMF}

FMF, the most common monogenic autoinflammatory disease, is caused by gain-of-function mutations in the MEFV gene. Most patients are of eastern Mediterranean descent, but FMF has also been reported in other populations. ${ }^{[22]}$ In the majority of patients, FMF is inherited in an autosomal recessive manner, but some mutations are recognized to cause dominant inheritance. Gain-of-function mutations result in decreased phosphorylation of pyrin, the protein encoded by $M E F V$, and lead to increased activation of the pyrin inflammasome and overproduction of IL-1 $13 .{ }^{[23]}$ Most patients present in childhood. FMF is characterized by recurrent and self-limiting episodes of fever lasting 1-3 days, abdominal and/or chest pain, mono-oligoarticular arthritis, and erysipelas-like rash. Long-term morbidity is mainly associated with renal amyloidosis. The most typical skin manifestation is erysipelas-like erythema, usually between the knee and the dorsum of the foot. The rash occurs more frequently in children and is associated with the most common and most severe mutation, M694V. ${ }^{\left[{ }^{9]}\right.}$ The lesion presents as a warm, tender, erythematous plaque with well-defined borders, usually $<15 \mathrm{~cm} .{ }^{[5]}$ The rash tends to subside within $24-48 \mathrm{~h}$ but may reoccur in the same place after long-distance walking. Histological examination demonstrates edema of the superficial dermis and sparse perivascular infiltrate composed of lymphocytes, neutrophils, and nuclear dust without vasculitis. Direct immunofluorescence showed deposits of $\mathrm{C} 3$ in the wall of the small vessels of the superficial vascular plexus, and fibrinogen and IgM in some cases. ${ }^{[24]}$

\section{PAAND}

PAAND is a dominantly inherited disease due to unique activating mutations in MEFV. ${ }^{[25]}$ Patients present in childhood with recurrent episodes lasting weeks and characterized by neutrophilic dermatosis, and also fever, myalgia, myositis, and sometimes abdominal pain. ${ }^{[1]}$ Neutrophilic dermatoses include severe pustular acne, sterile skin abscesses, hidradenitis suppurativa, neutrophilic panniculitis, small-vessel 
vasculitis, and pyoderma gangrenosum. Skin biopsy reveals predominantly neutrophilic, vascular, perivascular, and interstitial infiltrate. ${ }^{[25]}$ It has been proposed to use a general name, pyrin-associated autoinflammatory diseases (PAAD), to encompass all diseases associated with pyrin defects or MEFV mutations. ${ }^{[2]}$

\section{Haploinsufficiency of A20 (HA20)k}

HA20 is a recently discovered an autosomal dominant AID caused by mutations of the tumor necrosis factor alphainduced protein 3 (TNFAIP3, also known as A20,) gene, with early-onset and variable systemic inflammation. Pathogenic mutations in $\mathrm{A} 20$ (a negative regulator of NF-KB) result in reduced expression of the wild-type functional $A 20$, leading to increased activation of NF-KB in stimulated cells and thus enhanced release of cytokines including IL-1 $\beta$, TNF, IL-6, IL-18, and IL-17. ${ }^{[18]} A 20$ also regulates $B$ cell receptor and $T$ cell receptor signaling. Therefore, HA20 has a wide range of clinical presentations from Behçet's-like syndrome to autoinflammatory conditions such as systemic juvenile idiopathic arthritis (sJIA) and adult-onset Still's disease (AOSD), to classic autoimmune conditions including systemic lupus erythematosus (SLE) and immunodeficiency. ${ }^{[18]}$ First symptoms appear before 10 years of age in $73 \%$ of patients. ${ }^{[26]}$ Recurrent painful oral and/or genital ulcers reminiscent of Behçet's disease developed in $64 \%$ of patients. Cutaneous abscesses, erythema nodosum-like lesions (Figure 1E), acne, and folliculitis have also been reported. ${ }^{[5]}$

\section{Majeed Syndrome}

Majeed syndrome is a monogenic form of chronic nonbacterial osteomyelitis (CNO). ${ }^{[2]}$ It is caused by mutations in the lipin 2 (LPIN2) gene and is autosomal recessive inherited. Patients present with a triad of $\mathrm{CNO}$, neutrophilic dermatosis, and congenital dyserythropoietic anemia. Cutaneous manifestations include Sweet syndrome-like lesions, psoriasiform lesions, palmoplantar pustulosis, acne, and PG. ${ }^{[27]}$

\section{Autoinflammatory Granulomatosis}

Histopathologically, granulomatous dermatitis can be classified into 2 groups: necrobiotic/necrotizing and nonnecrobiotic/necrotizing. ${ }^{[28]}$ Cutaneous diseases in the latter group are characterized by the formation of "tight," nonnecrotizing/necrobiotic granulomas in the dermis, comprising histiocytes admixed with variable numbers of lymphoid cells.

\section{BS}

BS is one of the three phenotypes of nucleotide-binding oligomerization domain containing 2 (NOD2)-associated granulomatous disease, which also includes familial sarcoidosis and familial Crohn's disease. ${ }^{[2]}$ It is an autosomal dominant disease characterized by the clinical triad of granulomatous arthritis, uveitis, and dermatitis. ${ }^{[29]} \mathrm{BS}$ is caused by gain-of-function mutations in the NOD2 gene, likely through hyperactivation of NF-KB. Cutaneous manifestations of BS usually present early in life (before age 5 years) as the initial symptom. ${ }^{[1,5]}$ Typical lesions are small, erythematous nonconfluent papules, forming persistent desquamative rashes on the trunk and further onto the face and extremities, which gradually turn into brownish with a scaly appearance. ${ }^{[5]}$ Skin biopsies show non-caseating granulomas in the dermis, similar to sarcoidosis and Crohn's disease, with a variable number of lymphocytes and eosinophils. Other types of lesions have also been reported, including ichthyosiform dermatosis (Figure 1F), panniculitis mimicking erythema nodosum, livedoid lesions, leg ulcers, pityriasis lichenoides-like lesions, and leukocytoclastic vasculitis. ${ }^{[30]}$

\section{Autoinflammatory Chilblain}

Chilblain, also called pernio, is a superficial and localized inflammatory skin disorder that results from a maladaptive vascular response to non-freezing cold, which most commonly occurs on the toes, fingers, ears, and face. ${ }^{[31]}$ Chilblain lesions typically present as a painful and pruritic erythrocyanotic discoloration and swelling, persisting for $>24 \mathrm{~h}$. They usually resolve spontaneously in 1-3 weeks. Blisters, erosions, ulcerations, acrocyanosis, and Raynaud's phenomenon may be observed. Histopathologic findings of skin biopsy show dermal edema with superficial and deep perivascular lymphocytic infiltrate. The distribution of the infiltrate particularly around the eccrine gland is typical.

\section{Familial Chilblain Lupus}

Familial Chilblain Lupus, a type I interferonopathy, is autosomal-dominantly inherited and can be caused by several genetic defects in the process of type I interferon induction. Mutations in the following three genes have been identified to be responsible in different families: three prime repair exonuclease 1 (TREX1), SAM and HD domain containing deoxynucleoside triphosphate triphosphohydrolase 1 (SAMHD1), and stimulator of interferon response cGAMP interactor 1 (STING1). ${ }^{[32]}$ Familial Chilblain Lupus usually begins in early childhood and can lead to severe mutilations due to acral ischemia. ${ }^{[32]}$ Histological findings include a deep inflammatory infiltrate with perivascular distribution and granular deposits of immunoglobulins and complement along the basement membrane. ${ }^{[33]}$

\section{Aicardi-Goutieres Syndrome (AGS)}

AGS, another type I interferonopathy, is a hereditary neurodegenerative AID, characterized by early-onset progressive encephalopathy often leading to microcephaly, leukodystrophy, and basal ganglia calcification, accompanied by increased levels of IFNa and lymphocytosis in the cerebrospinal fluid. 
Clinical manifestations typically occur in infancy and disease progression can result in profound psychomotor retardation, and often death in early childhood. But asymptomatic individuals and delayed onset with the milder course are well recognized. This disease is classified into 7 types according to the causative gene: TREX1, RNASEH2B, RNASEH2C, RNASEH2A, SAMHD1, ADAR1, and IFIH1. Most of their encoded proteins have DNA/RNA degrading or modifying activities and their dysfunction leads to the accumulation of intracellular DNA/RNA. Because of excessive production of type I interferon, a proinflammatory state is developed leading to vasculopathy. ${ }^{[5]}$ The most severe neonatal form is typically due to mutations in the TREX1 gene. ${ }^{[4]}$ The appearance of chilblains upon cold exposure is an important clinical sign for correct diagnosis. Acrocyanosis, periungual erythema, and Raynaud's phenomenon can also be seen. Chilblain lesions develop as tender plaques on the feet, hands, ears, and nose, which spontaneously heal with possible hypopigmentation and atrophic scars. Histological findings include a dense lymphocytic infiltrate in the superficial dermis, and fibrinoid necrosis and thrombi within the lumen of the affected vessels. Interface dermatitis and basal vacuolar degeneration can be also present. Immunofluorescence studies and stains for mucin are both negative..$^{[5]}$

\section{SAVI}

SAVI is an autosomal dominant autoinflammatory vasculopathy caused by gain-of-function mutations in stimulator of interferon response cGAMP interactor 1 (STING1) gene, also known as transmembrane protein 173 (TMEM173) gene, leading to constitutive activation of the encoded protein, STING, and in turn production of type I interferons. ${ }^{[23]}$ SAVI is characterized by fever attacks, interstitial lung disease, and severe skin lesions on cold-sensitive areas. Although the vasculopathy of SAVI can resemble AGS, interstitial lung disease distinguishes it from most other interferonopathies. ${ }^{[1]}$ Cutaneous manifestations begin in infancy, mostly before 6 months of age. Patients suffer from erythematous purpuric patches and plaques on acral regions (fingers, toes, ears, and nose) giving rise to painful ulcerations with eschar formation, tissue infarction, and possible amputations. Dystrophic nail changes, nail fold capillary abnormalities, telangiectasia of the extremities, and hard palate have been reported. Erythematous plaques on cheeks mimicking malar rash are a characteristic feature of SAVI. Histological study shows dense neutrophilic infiltrates and karyorrhexis in the vessel walls, leukocytoclastic vasculitis, and microthrombotic angiopathy of small dermal vessels. ${ }^{[34]}$

\section{Autoinflammatory Lipodystrophy}

\section{PRAAS}

PRAAS, or more specifically proteasome subunit beta 8 (PSMB8)-PRAAS, ${ }^{[2]}$ is a spectrum of autosomal recessive
AIDs, caused by loss-of-function mutations of the PSMB8 gene. These syndromes include Nakajo Nishimura syndrome (NNS), joint contractures, muscular atrophy, microcytic anemia, panniculitis-induced lipodystrophy (JMP) syndrome, and chronic atypical neutrophilic dermatosis with lipodystrophy and elevated temperature (CANDLE) syndrome. JMP syndrome appears to be the most severe phenotype. ${ }^{[35]}$ PSMB8 mutations result in a pathologic reduction of proteasome activity leading to mitogen-activated protein kinase (MAPK) activation and subsequent upregulation of the interferon pathway and sustained inflammatory response. ${ }^{[36]}$ Patients of PRAAS typically present during infancy, and common clinical features are periodic fever, skin lesions, progressive lipodystrophy, muscular atrophy/ myositis, basal ganglia calcification, and hepatosplenomegaly. Pernio-like rash on ears, nose, hands, and feet, triggered by cold, appears early in life. Nodular erythema with infiltration and induration can also be observed. Another type of lesion is recurrent annular purpuric edematous plaques with raised borders and a flat center lasting for several days. Violaceous eyelid swelling is characteristic but typically seen in childhood and less visible after puberty. ${ }^{[5]}$ The common histological feature is a mixed perivascular and interstitial dense dermal infiltrate with predominantly CD 68+ mononuclear cells, histiocytes, eosinophils, and neutrophils, which often extends to the subcutaneous tissue. Panniculitis without vasculitis is reported in JMP and CANDLE syndromes. ${ }^{[35]}$ Progressive localized lipomuscular dystrophy especially in the face and upper body with elongated clubbed fingers is reported in all PRAAS cases. ${ }^{[13]}$ The pathogenesis of lipodystrophy is not fully understood, because mild to moderate panniculitis observed in skin biopsies from CANDLE patients can hardly explain the extensive fat loss in areas without skin lesions.

\section{ORAS}

ORAS, also known as Otulipenia, is an autosomal recessive disorder, caused by a loss-of-function mutation in the OTU deubiquitinase with linear linkage specificity (OTULIN) gene. ${ }^{[23]}$ This disorder is characterized by severe neonatal onset of recurrent fever, arthralgia, erythematous rashes with painful nodules, panniculitis, and lipodystrophy. Skin biopsies reveal neutrophilic dermatosis and predominantly septal panniculitis with vasculitis of small and medium-sized blood vessels. ${ }^{[5]}$ Lipoatrophy in ORAS is considered panniculitis-associated. ${ }^{[23]}$

\section{Autoinflammatory keratinization diseases}

Recently, AIDs showing psoriasis and related keratinization diseases have specifically been designated as "autoinflammatory keratinization diseases". ${ }^{[37]}$ In this group of AIDs, the main inflammation sites in the skin are the epidermis and the upper dermis and the inflammation leads to hyperkeratosis. 


\section{DITRA}

DITRA is an autosomal recessive AID, caused by loss-offunction mutations in the interleukin 36 receptor antagonist (IL36RN) gene. IL-36 receptor is expressed primarily in the skin and other epithelial cells in contact with the environment. ${ }^{[1]}$ Therefore, DITRA flares are featured by recurrent sudden-onset of severe generalized pustular psoriasis, usually with onset during childhood, accompanied by fever, malaise, and neutrophilia. ${ }^{[3]}$ Cutaneous manifestations present at first as diffuse and painful erythema and then turn into multiple small sterile pustules all over the body with scaly hyperkeratosis, and eventually coalesce to form large purulent collections. ${ }^{[5]}$ Interestingly, DITRA patients do not experience other types of psoriasis. ${ }^{[10]}$ Skin biopsy show intraepidermal spongiform pustules with clusters of neutrophils consolidated in microabscesses, and acanthosis and parakeratosis. ${ }^{[10]}$

\section{DIRA}

DIRA, an autosomal recessive disorder, is caused by lossof-function mutations in the interleukin 1 receptor antagonist $(I L 1 R N)$ gene and thus unopposed action at the IL-1 receptors. ${ }^{[9]}$ DIRA is characterized by a neonatal-onset severe pustular rash, multifocal sterile osteomyelitis, and periarticular soft-tissue swelling. ${ }^{[9]}$ Fever is usually absent. The severity of cutaneous presentation ranges from rare pustules to disseminated pustulosis. Skin pathergy may lead to the development of pustular lesions. Psoriatic nail changes, such as pitting and onychomadesis, may also be present. Skin biopsies have shown infiltration of the epidermis and dermis by neutrophils, formation of subcorneal pustules reminiscent of pustular psoriasis. ${ }^{[5]}$

\section{Cutaneous Manifestations of Other Main AIDs}

\section{MKD}

MKD, previously called hyperimmunoglobulin D syndrome (HIDS), is an autosomal recessive disorder caused by lossof-function mutations in the mevalonate kinase (MVK) gene. Mutated MVK results in the loss of pyrin inhibition. ${ }^{[23]}$ An associated disease, mevalonic aciduria (MVA), which is also caused by a mutation in the MVK gene, is now considered a severe phenotype of MKD. ${ }^{[2]}$ Patients with MKD present during infancy with fever, gastrointestinal symptoms, lymphadenopathy, arthralgia, myalgia, skin rash, and mucosal ulcers. ${ }^{[38]}$ Aphthous stomatitis is one of the three criteria in the Eurofever/PRINTO classification criteria for MKD, ${ }^{[7]}$ which is reported in $60 \%$ of patients. ${ }^{[38]}$ Cutaneous lesions include macules $(0.5-2.0 \mathrm{~cm})$ papules $(39 \%)$ on the limbs and trunk, urticarial lesions (15\%), cellulitis-like plaques, erythema elevatum diutinum, erythema nodosum, petechiae or purpura, and Sweet syndrome. ${ }^{[5]}$ A skin biopsy shows numerous polymorphonuclear neutrophils and few lymphocytes. ${ }^{[39]}$

\section{TRAPS}

TRAPS is a dominantly inherited recurrent fever disorder due to mutations in the TNF receptor superfamily member 1A (TNFRSF1A) gene, encoding TNF receptor type 1 . It is characterized by childhood-onset recurrent episodes often lasting 1 to 4 weeks. ${ }^{[1]}$ Common symptoms during attacks include fever, arthritis, myalgia, abdominal pain, headache, conjunctivitis, and periorbital edema. ${ }^{[0]}$ Cutaneous manifestation is various and present in 69-84\% during the attacks. ${ }^{[41]}$ Typical lesions include localized erythematous macules and papules (Figure 1G), edematous patches or plaques, and erysipelas-like rashes, commonly associated with underlying myalgias, which are migratory in a centrifugal pattern down the extremity in conjunction with the skin lesions. ${ }^{[42]}$ Migratory rash and periorbital edema are two criteria listed in the Eurofever/PRINTO classification criteria for TRAPS. ${ }^{[7]}$ Histologically, skin lesions in TRAPS are characterized by a perivascular dermal infiltrate of lymphocytes and monocytes without granulomatous or leukocytoclastic vasculitis. ${ }^{[42]}$

\section{DADA2}

DADA2 is an autosomal recessive multisystem disorder that features early-onset vasculitis resembling the medium vessel vasculitis polyarteritis nodosa, stroke, cytopenias, and immunodeficiency. ${ }^{[1]}$ DADA2 is caused by mutations in the adenosine deaminase 2 ( $A D A 2$ ) gene. The accumulation of adenosine due to mutated $A D A 2$ leads to uncontrolled activation of neutrophils. ${ }^{[43]}$ TNF appears to be the predominant driver of inflammation in DADA2 patients. ${ }^{[1]}$ Symptoms at presentation are often clinically indistinguishable from polyarteritis nodosa. Cutaneous manifestations include livedo reticularis/racemosa (Figure 1H), erythema nodosum, subcutaneous nodules, purpura, Raynaud's phenomenon, skin ulcers, and digital necrosis. Livedo racemosa is the most frequent, reported in $73 \%$ of patients. Skin biopsies show medium vessel vasculitis or leukocytoclastic vasculitis. ${ }^{[43]}$

\section{YAOS}

YAOS, formerly termed NOD2-associated autoinflammatory disease (NAID), is a polygenic AID characterized by recurrent fever, dermatitis, arthritis, swelling of the distal extremities, gastrointestinal and sicca-like symptoms with eyelid swelling. ${ }^{[4]}$ The disorder has a genetic association with certain NOD2 variants. ${ }^{[45]}$ Intermittent dermatitis develops in $90 \%$ of YAOS patients, which often manifested as erythematous patches, flushing, and plaques (Figure 1I). ${ }^{[46,47]}$ The rash predominantly affects the face, trunk, and limbs. It is mostly non-itchy or minimally itchy and occurs every few weeks to months with a duration of several days to weeks. Skin biopsies show spongiotic dermatitis in a majority of cases, and granulomatous dermatitis is extremely rare. ${ }^{[46]}$ 


\section{Conclusions}

The diagnosis of most AIDs requires not only a high index of suspicion and but also a characteristic phenotype to trigger a genetic sequencing. Although multiple systems are usually involved in AIDs, cutaneous lesions are often the first or prominent clinical manifestations of AIDs. Therefore, the recognition of cutaneous phenotypes is crucial for a correct diagnosis of AIDs. In this review, the cutaneous manifestations of $>20$ main AIDs were summarized. The phenotype-oriented classification used in this review also provides some clues to understand the common pathogenic pathways in which different AIDs lead to similar skin manifestations. Of note, the different categories in this classification are not mutually exclusive, and the cutaneous manifestations of one AID could definitely fall into multiple categories. The list of AIDs in this review is not exhaustive. However, using the classification as a framework, more novel AIDs could be organized based on their characteristic cutaneous manifestations to facilitate the diagnosis of AIDs in clinical practice.

Funding

This work was supported by the Natural Science Foundation of Beijing (Grant No. 7192170), the Chinese Academy of Medical Sciences Innovation Fund for Medical Sciences (CIFMS) (Grant No. 2017-I2M-3-001), and the National Key Research and Development Program of China (Grant No. 2016YFC0901500; 2016YFC0901501).

Conflict of Interest

Qingping Yao is an Editorial Board Member of the journal. The article was subject to the journal's standard procedures, with peer review handled independently of this member and his research groups.

\section{References}

[1] Nigrovic PA, Lee PY, Hoffman HM. Monogenic Autoinflammatory Disorders: Conceptual Overview, Phenotype, and Clinical Approach. J Allergy Clin Immunol, 2020;146(5):925-937.

[2] Ben-Chetrit E, Gattorno M, Gul A, et al. Consensus Proposal for Taxonomy and Definition of the Autoinflammatory Diseases (AIDs): A Delphi Study. Ann Rheum Dis, 2018;77(11):1558-1565.

[3] Krainer J, Siebenhandl S, Weinhausel A. Systemic Autoinflammatory Diseases. J Autoimmun, 2020;109:102421.

[4] Kanazawa N. Designation of Autoinflammatory Skin Manifestations With Specific Genetic Backgrounds. Front Immunol, 2020;11:475.

[5] Latour-Alvarez I, Torrelo A. Cutaneous Clues to Diagnose Autoinflammatory Diseases. G Ital Dermatol Venereol, 2020;155(5):551-566.

[6] Wu D, Shen M. Muckle-Wells Syndrome in Chinese Patients: A Single Center Case Series. Clin Rheumatol, 2017;36(4):965-969.

[7] Gattorno M, Hofer M, Federici S, et al. Classification Criteria for Autoinflammatory Recurrent Fevers. Ann Rheum Dis, 2019;78(8):1025-1032.

[8] Kuemmerle-Deschner JB. CAPS--pathogenesis, Presentation and Treatment of an Autoinflammatory Disease. Semin Immunopathol, 2015;37(4):377-385.

[9] Oldham J, Lachmann HJ. The Systemic Autoinflammatory Disorders for Dermatologists. Part 2: Disease Examples. Clin Exp Dermatol, 2020;45(8):967-973.

[10] Marzano AV, Damiani G, Genovese G, et al. A Dermatologic Perspective on Autoinflammatory Diseases. Clin Exp Rheumatol, 2018;36 Suppl 110:32-38.

[11] Shen M, Tang L, Shi X, et al. NLRP12 Autoinflammatory Disease: A Chinese Case Series and Literature Review. Clin Rheumatol, 2017;36(7):1661-1667.
[12] Wang W, Zhou Y, Zhong LQ, et al. The Clinical Phenotype and Genotype of NLRP12-Autoinflammatory Disease: A Chinese Case Series with Literature Review. World J Pediatrics, 2020;16(5): 514-519.

[13] Ombrello MJ, Remmers EF, Sun G, et al. Cold Urticaria, Immunodeficiency, and Autoimmunity Related to PLCG2 Deletions. N Engl J Med, 2012;366(4):330-338.

[14] Milner JD. PLAID: A Syndrome of Complex Patterns of Disease and Unique Phenotypes. J Clin Immunol, 2015;35(6):527-530.

[15] Shea J, Huynh T, Milner J, et al. PLAID Syndrome: Characteristic Presentation and a Novel Therapeutic Option. Pediatr Dermatol, 2020;37(1):147-149.

[16] Volker-Touw CM, de Koning HD, Giltay JC, et al. Erythematous Nodes, Urticarial Rash and Arthralgias in a Large Pedigree with NLRC4-related Autoinflammatory Disease, Expansion of the Phenotype. Br J Dermatol, 2017;176(1):244-248.

[17] Kitamura A, Sasaki Y, Abe T, et al. An Inherited Mutation in NLRC4 Causes Autoinflammation in Human and Mice. J Exp Med, 2014;211(12):2385-2396.

[18] Kacar M, Pathak S, Savic S. Hereditary Systemic Autoinflammatory Diseases and Schnitzler's Syndrome. Rheumatology (Oxford, England), 2019;58(Suppl 6):vi31-vi43.

[19] Yan R, Cao W, Liu X, et al. A Chinese Case Series of Schnitzler Syndrome and Complete Remission in One Tocilizumab-treated Patient. Clin Rheumatol, 2020;39(12):3847-3852.

[20] Gellrich FF, Gunther C. Schnitzler Syndrome. Hautarzt, 2019.

[21] Holzinger D, Roth J. Alarming Consequences - Autoinflammatory Disease Spectrum Due to Mutations in Proline-serine-threonine Phosphatase-interacting Protein 1. Curr Opin Rheumatol, 2016;28(5): 550-559. 
[22] Wu D, Shen M, Zeng X. Familial Mediterranean Fever in Chinese Adult Patients. Rheumatology (Oxford, England), 2018;57(12): 2140-2144.

[23] Savic S, Caseley EA, McDermott MF. Moving Towards a Systems-based Classification of Innate Immune-mediated Diseases. Nat Rev Rheumatol, 2020;16(4):222-237.

[24] Barzilai A, Langevitz P, Goldberg I, et al. Erysipelas-like Erythema of Familial Mediterranean Fever: Clinicopathologic Correlation. J Am Acad Dermatol, 2000;42(5):791-795.

[25] Masters SL, Lagou V, Jeru I, et al. Familial Autoinflammation with Neutrophilic Dermatosis Reveals a Regulatory Mechanism of Pyrin Activation. Sci Transl Med, 2016;8(332):332ra345.

[26] Yu MP, Xu XS, Zhou Q, et al. Haploinsufficiency of A20 (HA20): Updates on the Genetics, Phenotype, Pathogenesis and Treatment. World J Pediatrics, 2020;16(6):575-584.

[27] Moreira A, Torres B, Peruzzo J, et al. Skin Symptoms as Diagnostic Clue for Autoinflammatory Diseases. An Bras Dermatol,2017;92(1):72-80.

[28] Wick MR. Granulomatous \& Histiocytic Dermatitides. Semin Diagn Pathol 2017;34(3):301-311.

[29] Wu D, Shen M. Two Chinese Pedigrees of Blau Syndrome with Thirteen Affected Members. Clin Rheumatol, 2018;37(1):265-270.

[30] Bonnekoh H, Butze M, Kallinich T, et al. Spectrum of Genetic Autoinflammatory Diseases Presenting with Cutaneous Symptoms. Acta Derm Venereol, 2020;100(7):adv00091.

[31] Nyssen A, Benhadou F, Magnee M, et al. Chilblains. Vasa, 2020;49(2):133-140

[32] Fiehn C. Familial Chilblain Lupus - What Can We Learn from Type I Interferonopathies? Curr Rheumatol Rep, 2017;19(10):61.

[33] Lee-Kirsch MA, Gong M, Schulz H, et al. Familial Chilblain Lupus, A Monogenic Form of Cutaneous Lupus Erythematosus, Maps to Chromosome 3p. Am J Hum Genet, 2006;79(4): 731-737.

[34] Liu Y, Jesus AA, Marrero B, et al. Activated STING in a Vascular and Pulmonary Syndrome. N Engl J Med, 2014;371(6):507-518.

[35] Ohmura K. Nakajo-Nishimura Syndrome and Related Proteasome-associated Autoinflammatory Syndromes. J Inflamm Res, 2019;12:259-265.

[36] McDermott A, Jacks J, Kessler M, et al. Proteasome-associated Autoinflammatory Syndromes: Advances in Pathogeneses, Clinical
Presentations, Diagnosis, and Management. Int J Dermatol, 2015;54(2):121-129.

[37] Akiyama M, Takeichi T, McGrath JA, et al. Autoinflammatory Keratinization Diseases: An Emerging Concept Encompassing Various Inflammatory Keratinization Disorders of the Skin. J Dermatol Sci, 2018;90(2):105-111.

[38] Ter Haar NM, Jeyaratnam J, Lachmann HJ, et al. The Phenotype and Genotype of Mevalonate Kinase Deficiency: A Series of 114 Cases From the Eurofever Registry. Arthritis Rheumatol, 2016;68(11):2795-2805.

[39] Drenth JP, Boom BW, Toonstra J, et al. Cutaneous Manifestations and Histologic Findings in the Hyperimmunoglobulinemia D Syndrome. International Hyper IgD Study Group. Arch Dermatol, 1994;130(1):59-65.

[40] Zhao M, Luo Y, Wu D, et al. Clinical and Genetic Features of Chinese Adult Patients with Tumour Necrosis Factor Receptorassociated Periodic Fever Syndrome. Rheumatology (Oxford, England), 2020;59(8):1969-1974.

[41] Yao Q, Englund KA, Hayden SP, et al. Tumor Necrosis Factor Receptor Associated Periodic Fever Syndrome with Photographic Evidence of Various Skin Disease and Unusual Phenotypes: Case Report and Literature Review. Semin Arthritis Rheum, 2012;41(4):611-617.

[42] Schmaltz R, Vogt T, Reichrath J. Skin Manifestations in Tumor Necrosis Factor Receptor-associated Periodic Syndrome (TRAPS). Dermato-endocrinology, 2010;2(1):26-29.

[43] Kendall JL, Springer JM. The Many Faces of a Monogenic Autoinflammatory Disease: Adenosine Deaminase 2 Deficiency. Curr Rheumatol Rep, 2020;22(10):64.

[44] Yang X, Wu D, Li J, et al. A Chinese Case Series of Yao Syndrome and Literature Review. Clin Rheumatol, 2018;37(12):3449-3454.

[45] Yao Q, Kontzias A. Expansion of Phenotypic and Genotypic Spectrum in Yao Syndrome: A Case Series. J Clin Rheumatol, 2020; Publish Ahead of Print.

[46] Yao Q, Shen M, McDonald C, et al. NOD2-associated Autoinflammatory Disease: A Large Cohort Study. Rheumatology (Oxford, England), 2015;54(10):1904-1912.

[47] Yao Q, Shen B. A Systematic Analysis of Treatment and Outcomes of NOD2-Associated Autoinflammatory Disease. Am J Med, 2017;130(3):365 e313-365 e318. 